\title{
Acute Esophageal Necrosis Secondary to a Paraesophageal Hernia
}

\author{
Chris J. Lia Benjamin B. Claxton ${ }^{a}$ Peter Block ${ }^{a}$ Sean Reilly ${ }^{a}$ \\ Scott Manski ${ }^{b}$ Cuckoo Choudhary ${ }^{b}$ \\ aDepartment of Internal Medicine, Thomas Jefferson University Hospitals, Philadelphia, PA,

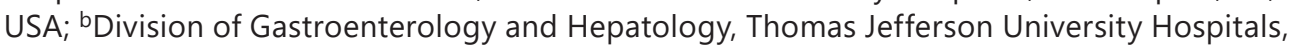 \\ Philadelphia, PA, USA
}

\section{Keywords}

Acute esophageal necrosis · Black esophagus · Paraesophageal hernia · Hematemesis

\begin{abstract}
Acute esophageal necrosis (AEN) or "black esophagus" is a rare clinical entity caused by necrosis of distal esophageal mucosa stemming from esophageal ischemia. Possible etiologies are broad but most commonly include possible triggers of low-flow vascular states in the esophagus, including infections, broad-spectrum antibiotic use, and gastric volvulus, among others. Patients most commonly present clinically with acute onset hematemesis and melena. Here, we describe a patient who initially presented with multiple nonspecific gastrointestinal symptoms, including abdominal pain and nausea, that progressed over a 10-day period, culminating in multiple episodes of hematemesis prior to presentation. Endoscopic evaluation confirmed the diagnosis of AEN and unveiled a possible paraesophageal hernia (PEH) as the causative factor. A subsequent videofluoroscopic barium swallow was utilized to better characterize the upper gastrointestinal anatomy and confirmed the PEH as a likely etiology. Esophagogastroduodenoscopy (EGD) can often identify PEH independently, but in patients with AEN secondary to a possible, but unclear, PEH on EGD, a videofluoroscopic barium swallow is an appropriate and useful next step in confirming the diagnosis. While treatment of AEN traditionally involves fluid resuscitation, intravenous protein pump inhibitors, and total parenteral nutrition, surgical intervention is often indicated in patients who have a contributing and symptomatic PEH.
\end{abstract}

\section{Karger"}




\section{Introduction}

Acute esophageal necrosis (AEN), also known as "black esophagus," is a rare clinical finding characterized by diffuse necrosis of the distal esophagus, typically terminating at the gastroesophageal junction [1]. Clinically, $70 \%$ of patients present with acute onset hematemesis and melena. Less common presentations include dysphagia, chest pain, epigastric pain, and fever [2]. It occurs due to esophageal ischemia, secondary to either a low flow state of the esophagus from a multitude of inciting factors or disruption of the mucosal barrier system, which is seen in debilitated patients. Here, we present a case of acute esophageal necrosis associated with a PEH.

\section{Case Report}

A 60 year-old-woman with a history of daily alcohol use, osteoarthritis on chronic nonsteroidal anti-inflammatory drugs (NSAIDs), and paroxysmal supraventricular tachycardia presented to the emergency department with a 10-day history of abdominal pain, nausea, and emesis. Initially nonbloody, the patient reported several episodes of hematemesis 1 day prior to admission. This was associated with persistent, nonexertional, substernal chest pain, without radiation. She reported associated anorexia. The patient denied any recent NSAID, alcohol, or foreign substance ingestion.

On arrival to the emergency department, the patient was tachycardic to 148 beats per minute and hypotensive to $103 / 92$, with otherwise stable vital signs. Her labs revealed an elevated serum creatinine of $1.81 \mathrm{mg} / \mathrm{dL}$ from a baseline of $0.6 \mathrm{mg} / \mathrm{dL}$, serum lactate of 4 $\mathrm{mmol} / \mathrm{L}$, and an elevated anion gap of $37 \mathrm{mmol} / \mathrm{L}$. After aggressive intravenous fluid resuscitation, these lab abnormalities normalized. Labs also revealed a serum white blood cell count of 24,000 B/L, hemoglobin of $13.5 \mathrm{~g} / \mathrm{dL}$, and platelets of 301,000 B/L. High-sensitivity troponin was elevated $(240 \rightarrow 217 \mathrm{ng} / \mathrm{mL})$. An electrocardiogram revealed atrial fibrillation with rapid ventricular response without evidence of acute coronary syndrome. Chest radiography revealed mild pulmonary edema and was otherwise unremarkable. She was started on an intravenous proton pump inhibitor.

Upper endoscopy showed hemorrhagic mucosa at $20 \mathrm{~cm}$ from the incisors, which became necrotic circumferentially, extending to the esophagogastric junction (shown in Fig. 1). A large hiatal hernia was also appreciated. No esophageal varices were seen. She received a 4-day course of empiric antimicrobials for potential microbial translocation, was placed on bowel rest, and was started on total parenteral nutrition. She was discharged 6 days after presentation.

The patient underwent an outpatient esophagogastroduodenoscopy (EGD) 1 month later, which demonstrated improving esophagitis and a large hiatal hernia, but was inconclusive for PEH. Subsequent videofluoroscopic barium swallow revealed a moderate-sized fixed type II PEH, defined by a normal anatomically placed gastroesophageal junction with a portion of the gastric fundus herniating through the diaphragmatic hiatus adjacent to the esophagus. The patient was referred for surgical management.

\section{Discussion}

The pathogenesis of acute esophageal necrosis is theorized to commonly stem from ischemic insults or gastric outlet obstruction. Low flow vascular states as well as esophageal reflux both promote a direct injury of the esophageal mucosa [3]. The distal third of the 

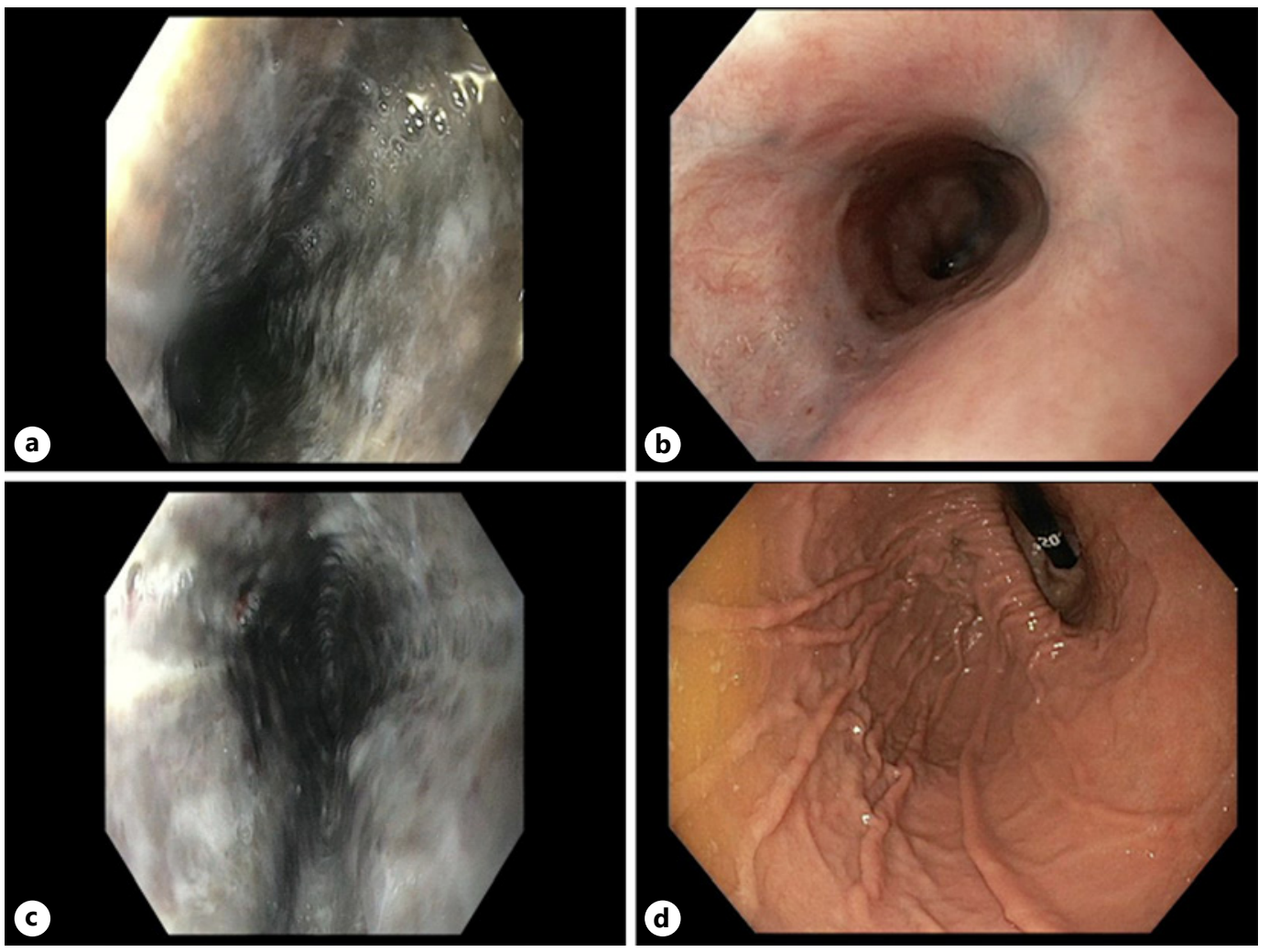

Fig. 1. a, c Endoscopic findings from admission, demonstrating acute, circumferential necrosis of the esophagus extending to the esophagogastric junction. b, Endoscopic findings about 30 days after admission, revealing mucosal healing. $d$, A retroflexed endoscopic view in the stomach showing the paraesophageal hernia.

esophagus is a "watershed" area relative to the densely vascularized upper esophagus, receiving blood from branches of the left gastric and inferior phrenic arteries. Despite the relatively hypovascular nature of the distal esophagus, the complex vascular network in the esophageal submucosa makes esophageal infarction a rare clinical entity [4]. First-line conservative treatment modalities are focused on restoring flow and suppressing reflux with intravenous fluids, proton pump inhibitors, total parenteral nutrition, and minimal oral intake.

The inciting event causing esophageal ischemia is thought to be multifactorial; here, we postulate that transient torsion of the esophagus in the presence of a PEH was the inciting factor of esophageal injury. This has been described infrequently in the literature, with similar presentations of abdominal pain and hematemesis [5]. In patients with AEN secondary to $\mathrm{PEH}$, resolution of symptoms has traditionally been achieved with urgent surgical intervention for hernia repair, volvulus reduction, and incarceration of the gastric antrum. With an overall mortality rate of $32 \%$, an early recognition of AEN and initiation of treatment are imperative to prevent rapid deterioration [6]. Her past history of NSAID and alcohol use is an unlikely etiology given the risk of upper gastrointestinal bleeds is decreased in chronic NSAID use (when compared to acute), and typical imaging findings of bleeding secondary to both substances were absent on EGD [7].

In patients with AEN thought be secondary to a PEH without confirmatory findings on EGD, videofluoroscopic barium swallow may be utilized to better characterize the upper gastrointestinal anatomy [8]. Patients with a PEH may be managed with supportive care, which can improve esophageal necrosis prior to definitive surgical intervention. Specific indications for surgery in 
a PEH include symptomatic presentations, particularly in those with acute obstructive symptoms who have undergone volvulus [9]. AEN is a rare but important clinical finding in a patient with hematemesis with a high mortality rate that requires urgent and aggressive therapy.

\section{Statement of Ethics}

Written informed consent was obtained from the patient for publication of this case report and any accompanying images. Information revealing the subject's identity was avoided. Ethical approval was not required in accordance with Jefferson Office of Human Research.

\section{Conflict of Interest Statement}

All authors have no declared or potential conflicts of interests that may compromise the design and conduct of the study, collection, analysis and interpretation of the data, and the preparation, review, or approval of the manuscript. Cuckoo Choudhary has had full access to all the data in the study and takes responsibility for the integrity of the data.

\section{Funding Sources}

No funding sources, sponsors, or other financial entities were relevant to the study.

\section{Author Contributions}

Chris Li's contribution consisted of conception and design, drafting of the manuscript, and critical revision of the manuscript. Benjamin Claxton, Peter Block, and Sean Reilly's contributions consisted of analysis and interpretation of data, drafting the manuscript, and critical revision of the manuscript for important intellectual content. Scott Manski and Cuckoo Choudhary provided analysis and interpretation of the data, critical revision of the manuscript for important intellectual content, and final approval of the manuscript.

\section{References}

1 Geller A, Aguilar H, Burgart L, Gostout CJ. The black esophagus. Am J Gastroenterol. 1995;90(12):2210-2.

2 Gurvits GE, Shapsis A, Lau N, Gualtieri N, Robilotti JG. Acute esophageal necrosis: a rare syndrome. J Gastroenterol. 2007;42(1):29-38.

3 Jacobsen NO, Christiansen J, Kruse A. Incidence of oesophageal necrosis in an autopsy material. APMIS. 2003; 111(5):591-4.

4 Gurvits GE. Black esophagus: acute esophageal necrosis syndrome. World J Gastroenterol. 2010;16(26):3219-25.

5 Nunes G, Patita M, Fernandes V, Fonseca J. Paraesophageal hernia and gastric volvulus: an uncommon etiology of vomiting and upper gastrointestinal bleeding. Rev Esp Enferm Dig. 2017;109(4):294-5.

6 Neumann DA, Francis DL, Baron TH. Proximal black esophagus: a case report and review of the literature. Gastrointest Endosc. 2009;70(1):180-1.

7 Pilotto A, Franceschi M, Leandro G, Paris F, Niro V, Longo MG, et al. The risk of upper gastrointestinal bleeding in elderly users of aspirin and other non-steroidal anti-inflammatory drugs: the role of gastroprotective drugs. Aging Clin Exp Res. 2003 Dec;15(6):494-9.

8 Weitzendorfer M, Köhler G, Antoniou SA, Pallwein-Prettner L, Manzenreiter L, Schredl P, et al. Preoperative diagnosis of hiatal hernia: barium swallow X-ray, high-resolution manometry, or endoscopy? Eur Surg. 2017;49(5):210-7.

9 Kohn GP, Price RR, DeMeester SR, Zehetner J, Muensterer OJ, Awad Z, et al. Guidelines for the management of hiatal hernia. Surg Endosc. 2013 Dec;27:4409-28. 